\title{
Cobalt Oxyhydroxide-prompted Synthesis of Fluorescent Polydopamine Nanoparticles for Glutathione Detection
}

\author{
QiaoQin Yu, YanYan ZhaO, WenJing Deng, TingTing Chen, ${ }^{\dagger}$ and Xia $\mathrm{CHU}^{\dagger}$ \\ State Key Laboratory of Chemo/Bio-Sensing and Chemometrics, College of Chemistry and Chemical Engineering, \\ Hunan University, Changsha 410082, P. R. China
}

\begin{abstract}
Glutathione (GSH) plays an important role in cells, which is an essential endogenous antioxidant. Here, we have developed a new detection platform to analyze GSH levels. In our system, fluorescent polydopamine (PDA) nanoparticles, as signal indicators, were obtained by oxidation through cobalt oxyhydroxide $(\mathrm{CoOOH})$ nanosheets. When $\mathrm{CoOOH}$ was present, $\mathrm{CoOOH}$ could quickly oxidize dopamine to fluorescent PDA nanoparticles. However, once GSH existed, $\mathrm{CoOOH}$ nanosheets were decomposed into $\mathrm{Co}^{2+}$, and oxidation between $\mathrm{CoOOH}$ and dopamine was prevented with weaker fluorescence occurring. Thus, we could realize detection of the GSH concentration according to the decreased fluorescence value of the fluorescent polydopamine. This method provides a fast, simple, high sensitivity and desirable selectivity platform for GSH monitoring.
\end{abstract}

Keywords Cobalt oxyhydroxide $(\mathrm{CoOOH})$ nanosheets, dopamine, oxidation-reduction reaction, fluorescent polydopamine nanoparticles, glutathione (GSH)

(Received September 11, 2019; Accepted October 15, 2019; Advance Publication Released Online by J-STAGE October 25, 2019)

\section{Introduction}

Glutathione (GSH) is the most abundant thiolated tripeptide present in life species. GSH is an important endogenous antioxidant that prevents toxins and free radicals in cells. ${ }^{1}$ GSH levels are often associated with many diseases. ${ }^{2,3}$ The results of the study showed that GSH concentration decreased in patients with liver disease, diabetes, and cataract. ${ }^{4,5}$ Chinta et al. found that patients with Parkinson's disease and Alzheimer's disease also had lower GSH concentrations. ${ }^{6}$ In addition, an abnormal content of GSH is also related to the body mass index. ${ }^{7}$ Therefore, it is very important to monitor the GSH concentration. To date, various methods for measuring GSH have been developed, including fluorescence-electroanalysis (FL-EC), ${ }^{8}$ liquid chromatography (LC), ${ }^{9}$ liquid chromatography-mass spectrometry (LC-MS), ${ }^{10}$ capillary electrophoresis (CE), ${ }^{11}$ electroanalysis (EC), ${ }^{12}$ and electrochemiluminescence (ECL). ${ }^{13}$ Conventional methods for detecting the cellular GSH concentration typically utilize thiol-sensitive organic fluorophores. ${ }^{14,15}$ Despite their usefulness, the organic fluorophores cannot be measured for long periods because of photobleaching and sample labeling at large depths due to lowdepth tissue penetration. Therefore, it is necessary to find a simple, fast, sensitive and low-cost method to monitor the level of GSH.

Fluorescent nanoparticles are receiving increasing attention concerning their potential applications in labeling, sensing and biomedicine fields. ${ }^{16-22}$ Compared with traditional organic

$\dagger$ To whom correspondence should be addressed.

E-mail: xiachu@hnu.edu.cn (X. C.); chenting1104@hnu.edu.cn (T. T. C.) fluorescent dyes, fluorescent nanoparticles e.g. quantum dots, polymer nanoparticles and upconverting nanoparticles, have unique optical and chemical performances, for example, high fluorescence intensity, strong light stability and great biocompatibility. ${ }^{23-29}$ In particular, polymeric nanoparticles have become a promising material own to strong stability in the environment and better controllable surface properties. ${ }^{30}$

Here, we have developed a new simple, sensitive and fast sensing method based on polymer fluorescent nanoparticles for GSH detection. As an important neurotransmitter in the human brain, dopamine can regulate various biological functions. Dopamines' catechol is easily oxidized to an anthracene derivative under oxidative conditions, and can be selfpolymerized into fluorescent polydopamine nanoparticles. Based on this property of dopamine, endogenous fluorescent PDA nanoparticles with cobalt oxyhydroxide $(\mathrm{CoOOH})$ as an oxidant was successfully prepared, and further constructed a platform based on $\mathrm{CoOOH}$ and GSH redox reactions to detect GSH. When no GSH was present, $\mathrm{CoOOH}$ could oxidize dopamine to fluorescent polydopamine particles. But once the $\mathrm{CoOOH}$ in the system was firstly reacted with GSH, GSH could reduce it to $\mathrm{Co}^{2+}$ with no oxidation ability, resulting in the inability to continue to oxidize dopamine to produce fluorescence. In the system that we developed, the signal indicator was the fluorescent polydopamine particle. The concentration of GSH could be detected by observing changes in the particle's fluorescence. This sensing method exhibited great detection capability in a GSH assay, and its detection limit was as low as $1.93 \mu \mathrm{M}$. This method had a desirable selectivity for GSH compared to other potentially interfering substances. In addition, this method showed practical application in GSH analysis in human blood samples, and provided a meaningful detection means in clinical diagnosis and biological detection. 


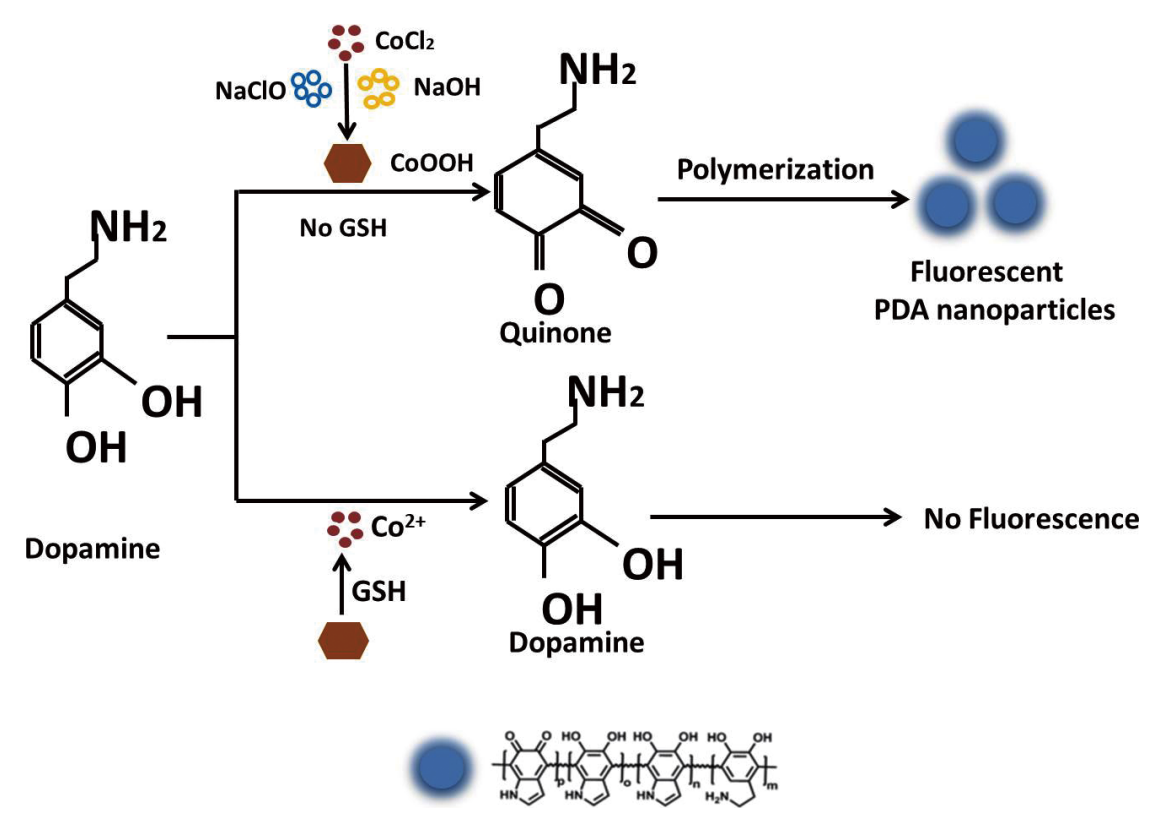

Scheme 1 Principle of the fluorescent polydopamine particle-based strategy for GSH detecting.

\section{Experimental}

\section{Reagents and materials}

Cobaltous chloride $\left(\mathrm{CoCl}_{2} \cdot 6 \mathrm{H}_{2} \mathrm{O}\right)$, sodium hypochlorite $(\mathrm{NaClO})$, sodium hydroxide $(\mathrm{NaOH})$, sodium chloride $(\mathrm{NaCl})$, magnesium chloride $\left(\mathrm{MgCl}_{2}\right)$, potassium chloride $(\mathrm{KCl})$, calcium chloride $\left(\mathrm{CaCl}_{2}\right)$, hydrochloric acid, all amino acids and glucose, were purchased from China National Pharmaceutical Group Corp. Dopamine, vitamin C and L-glutathione (GSH) were provided by Sigma-Aldrich. YSL (composed of tyrosine, serine and leucine), YSV (composed of tyrosine, serine and valine) was obtained from Kangzhe Pharmaceutical (Shenzhen, China). RGD (composed of arginine, glycine and aspartic acid) was provided by Puzhen Organism (Shanghai, China). The First Xiangya Hospital (Changsha, China) provided human blood. All solutions were prepared by an electric resistance of $>18.2 \mathrm{M} \Omega$ ultrapure water, which was obtained via a Millipore Milli-Q water purification system (Billerica, MA, USA).

\section{Apparatus}

Fluorescence spectra were from an F-7000 fluorescence spectrometer (HORIBA, Japan). The scanning range was collected in the range of $430-600 \mathrm{~nm}$ and its excitation wavelength was $390 \mathrm{~nm}$. TEM images were acquired by a fieldemission high-resolution 2100F TEM (JEOL, Japan) manipulating at a $200-\mathrm{kV}$ acceleration voltage. The UV-vis absorption spectrum was obtained with a UV-2450 UV-visible spectrometer (Shimadzu, Japan).

\section{Preparation of $\mathrm{CoOOH}$}

$\mathrm{CoOOH}$ nanosheets were performed by the previously reported method with some modifications. ${ }^{1}$ Briefly, a $500-\mu \mathrm{L}$ $10 \mathrm{mM} \mathrm{CoCl} 2 \cdot 6 \mathrm{H}_{2} \mathrm{O}$ solution was added into $125 \mu \mathrm{L}$ of $1.0 \mathrm{M}$ $\mathrm{NaOH}$ and ultrasonicated for $60 \mathrm{~s}$. Then, $25 \mu \mathrm{L}$ of $0.9 \mathrm{M}$ $\mathrm{NaClO}$ was added and reacted 30 min under ultrasonication at $25^{\circ} \mathrm{C}$. The final solution was centrifuged at $13000 \mathrm{rpm}$ for $30 \mathrm{~min}$ and was continuously washed four times with sterilized water. Lastly, we dispersed the nanosheets into $1 \mathrm{~mL}$ of sterile water and placed at $4^{\circ} \mathrm{C}$ for use. The final concentration of $\mathrm{CoOOH}$ was about $0.75 \mathrm{mg} \mathrm{mL}^{-1}$.

Preparation of fluorescent polydopamine

To obtained fluorescent PDA particles, $86 \mu \mathrm{L}$ of water, $6 \mu \mathrm{L}$ of $0.75 \mathrm{mg} \mathrm{mL}^{-1} \mathrm{CoOOH}$ nanosheets and $5 \mu \mathrm{L}$ of a $25 \mathrm{mM}$ dopamine solution were mixed in a $200-\mu \mathrm{L}$ PCR tube, and then the mixed solution was incubated for $40 \mathrm{~min}$ at $25^{\circ} \mathrm{C}$. Finally, $3 \mu \mathrm{L}$ of $0.2 \mathrm{M} \mathrm{HCl}$ was added to regulate the $\mathrm{pH}$.

\section{GSH detection}

Firstly, $6 \mu \mathrm{L}$ of $0.75 \mathrm{mg} \mathrm{mL}^{-1} \mathrm{CoOOH}$ nanosheets was incubated with different concentrations of L-glutathione for $20 \mathrm{~min}$ at room temperature. Then, $5 \mu \mathrm{L}$ of a $25 \mathrm{mM}$ dopamine was put into the system and reacted for $40 \mathrm{~min}$ at $25^{\circ} \mathrm{C}$ to obtain fluorescent PDA. Ultimately, $3 \mu \mathrm{L}$ of $0.2 \mathrm{M} \mathrm{HCl}$ was introduced into the above mixture to stop the reaction.

To test GSH in whole blood, heparin was first used for an anticoagulant treatment. Briefly, $200 \mu \mathrm{L}$ of whole blood was treated by $5 \mu \mathrm{L}$ of a $100 \mathrm{mM} \mathrm{L}$-serine-borate complex to inhibit the gamma-glutamyltranspeptidase activity and then the mixture was centrifuged for $15 \mathrm{~min}$ at $12000 \mathrm{rpm}$ to remove haemocytes. Finally, the supernatant fluid was obtained. After supernatant fluid was diluted 50 times, it was immediately introduced into the sensing platform and other operations were similar to the above.

\section{Results and Discussion}

Principle of preparing fluorescent polydopamine particles and detecting $G S H$

Firstly, according to previous literature reports, $\mathrm{CoOOH}$ nanosheets were simply synthesized using $\mathrm{CoCl}_{2}, \mathrm{NaClO}$, and $\mathrm{NaOH} .{ }^{31}$ When dopamine was mixed with $\mathrm{CoOOH}$ nanosheets, dopamine could be oxidized by $\mathrm{CoOOH}$ to an anthracene derivative. The anthracene derivative could then be spontaneously polymerized into fluorescent polydopamine nanoparticles. Based on this, we designed a new platform to 

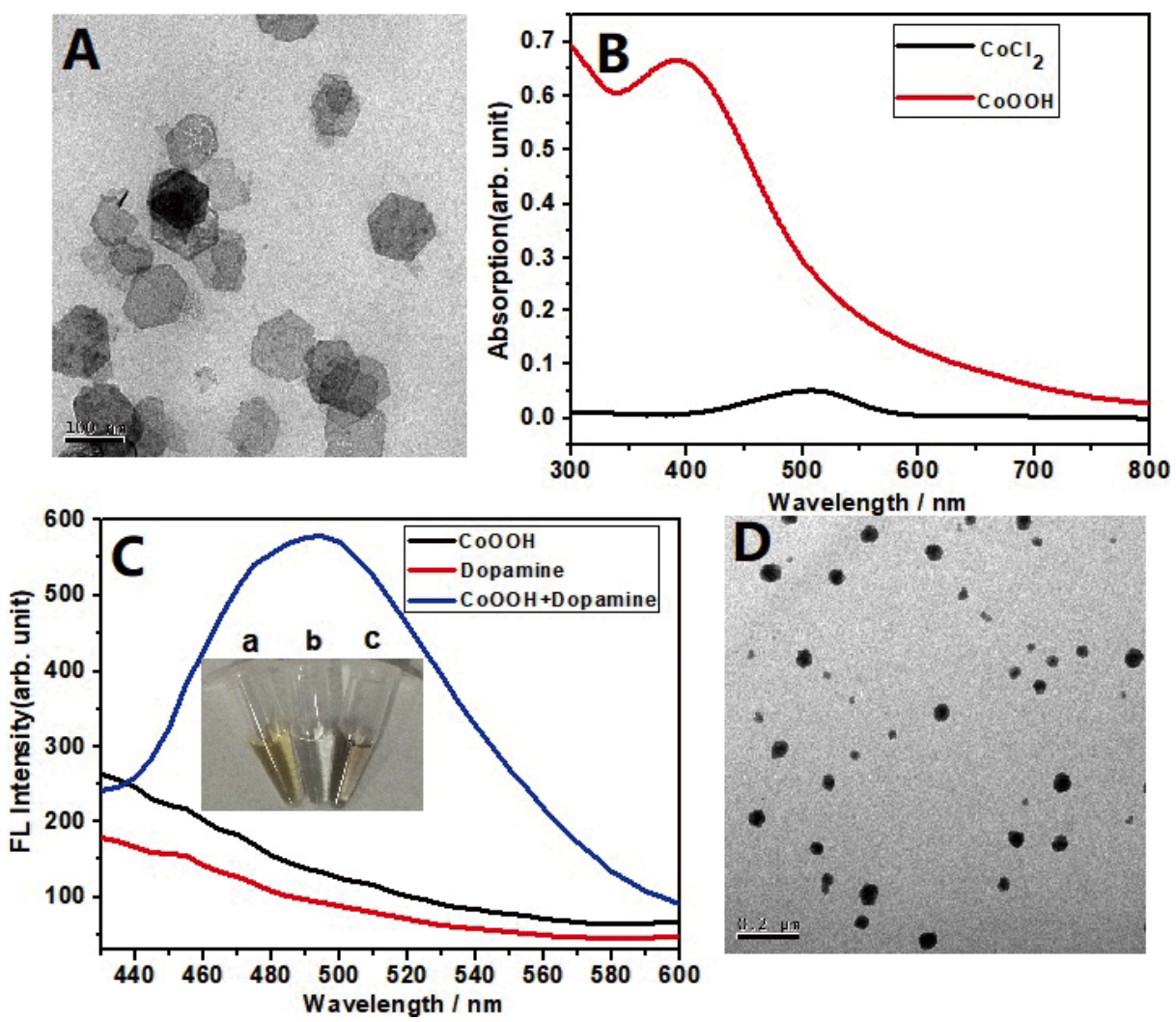

Fig. 1 (A) TEM image of the prepared CoOOH. Scale bars: $100 \mathrm{~nm}$. (B) UV-vis absorption spectrum of the $\mathrm{CoOOH}$ nanosheet (red line) and $\mathrm{CoCl}_{2}$ (black line). (C) Fluorescence emission spectra of $1.25 \mathrm{mM}$ dopamine (red line), $45 \mu \mathrm{g} \mathrm{mL} \mathrm{m}^{-1} \mathrm{CoOOH}$ (black line) and $45 \mu \mathrm{g} \mathrm{mL}^{-1} \mathrm{CoOOH}+1.25 \mathrm{mM}$ dopamine (blue line). Iconography: photographs of $45 \mu \mathrm{g} \mathrm{mL}^{-1} \mathrm{CoOOH}$ (tube a), $1.25 \mathrm{mM}$ dopamine (tube b) and the mixture of $1.25 \mathrm{mM}$ dopamine and $45 \mu \mathrm{g} \mathrm{mL}-1 \mathrm{CoOOH}$ (tube c). (D) TEM image of the obtained fluorescent polydopamine particles. Scale bars: $0.2 \mu \mathrm{m}$.

detect GSH. The detection principle is shown in Scheme 1. In the presence of $\mathrm{GSH}, \mathrm{CoOOH}$ was decomposed to $\mathrm{Co}^{2+}$. The reaction equation was as follows:

$$
2 \mathrm{CoOOH}+2 \mathrm{GSH}+4 \mathrm{H}^{+} \longrightarrow 2 \mathrm{Co}^{2+}+\mathrm{GS}-\mathrm{SG}+4 \mathrm{H}_{2} \mathrm{O}
$$

Therefore, the fluorescent polydopamine nanoparticles were not synthesized because $\mathrm{CoOOH}$ was decomposed so that the oxidation for dopamine cannot be achieved. Conversely, when there was no $\mathrm{GSH}, \mathrm{CoOOH}$ nanosheets were retained in solution and dopamine would be oxidized to quinone. We then obtained fluorescent PDA by the self-polymerization of quinone. Accordingly, the detection of GSH was achieved by observing the decrease in the fluorescence signal of fluorescent polydopamine particles.

\section{Charactering $\mathrm{CoOOH}$ and fluorescent polydopamine particles}

Three characterization methods, including transmission electron microscope (TEM), energy dispersive X-ray spectrometer (EDS) and UV-vis absorption spectra, were used to characterize as-synthesized $\mathrm{CoOOH}$ nanosheets. As shown in Fig. 1A, the TEM result certified that the newly prepared $\mathrm{CoOOH}$ presented a hexagonal morphology. The $\mathrm{O}$ and Co elements were found in $\mathrm{CoOOH}$ nanosheets from EDS analysis (Fig. S1, Supporting Information). We also evaluated the stability of $\mathrm{CoOOH}$ nanosheets in water for over seven days (Fig. S2, SI). It could be seen from TEM images that $\mathrm{CoOOH}$ still maintained a hexagonal morphology, which proved that $\mathrm{CoOOH}$ was stable.
Furthermore, the optical performance of $\mathrm{CoOOH}$ was ensured by UV-vis absorption. As can be seen in Fig. 1B, the absorbance spectrum displayed a peak centered at $410 \mathrm{~nm}$. These results displayed the successful synthesis of $\mathrm{CoOOH}$ nanosheets. ${ }^{31,32}$ Figure $1 \mathrm{C}$ affirmed that the fluorescent PDA nanoparticles were successfully prepared. When dopamine was mixed with $\mathrm{CoOOH}$ (blue line) we could see a remarkable fluorescence signal, while no obvious fluorescence signal was generated when there was only $\mathrm{CoOOH}$ or dopamine. The obtained fluorescent PDA particles had an irregular shape and different size of about $10-50 \mathrm{~nm}$ according to TEM image from Fig. 1D because of the stacking conditions of oligomers and different oligomeric units. ${ }^{33,34}$ In Fig. S3A (SI), the excitation wavelength of fluorescent PDA particles was $390 \mathrm{~nm}$ and the maximum emission wavelength was $490 \mathrm{~nm}$. From Fig. S3B, we could find that the emission intensity showed an increasing trend at between 360 and $390 \mathrm{~nm}$ and then began to decrease in the range of $390-410 \mathrm{~nm}$. When the excitation wavelength was constantly changing, the position of the emission peak hardly changed. This might be related to the mild acidic reaction conditions. ${ }^{34}$ Moreover, we also tested the change in the fluorescence value of fluorescent polydopamine particles for over seven days. In Fig. S4 (SI), the intensity of the fluorescent polydopamine particles remained unchanged, illustrating that the fluorescent polydopamine particles were stable.

\section{Feasibility analysis}

At first, we used the changes of the fluorescence value to 


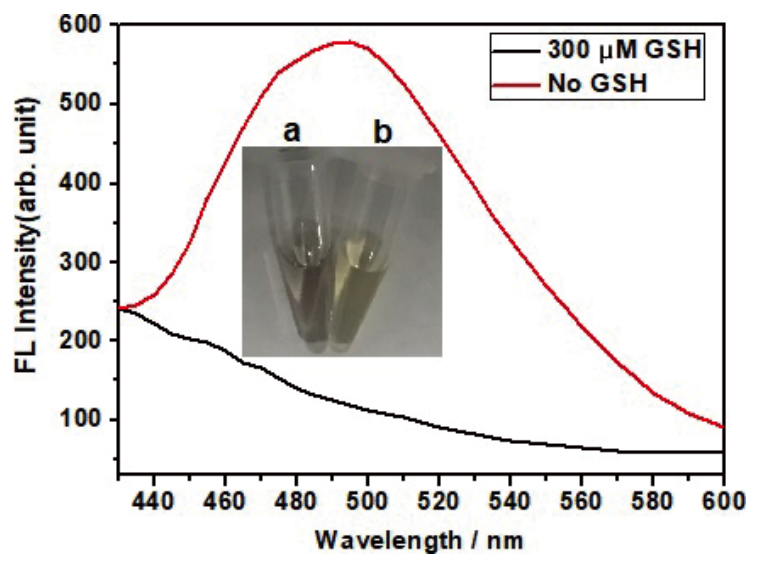

Fig. 2 Fluorescence emission spectrum of fluorescent polydopamine particles with $300 \mu \mathrm{M}$ GSH (black line) and without GSH (red line). Iconography: photographs of the prepared fluorescent PDA particles without GSH (tube a) and with $300 \mu \mathrm{M} \mathrm{GSH}$ (tube b).

verify the feasibility of the proposed sensing strategy (Fig. 2). When GSH was not present (red line), the synthesized fluorescent PDA nanoparticles had strong fluorescence at $490 \mathrm{~nm}$, while when GSH was existent in the system (black line), the fluorescence intensity of PDA was significantly reduced, thus demonstrating that $\mathrm{GSH}$ could reduce $\mathrm{CoOOH}$ to $\mathrm{Co}^{2+}$ and inhibit the production of fluorescent PDA nanoparticles. In addition, we also tested the UV-vis absorption spectrum to demonstrate the feasibility of this principle (Fig. S5, SI). When GSH was present (green line), $\mathrm{CoOOH}$ was reduced and could not continue to oxidize dopamine. The absorption spectrum was similar to the same concentration of dopamine solution (red line) and the peak at $410 \mathrm{~nm}$ of $\mathrm{CoOOH}$ (black line) disappeared On the contrary, when GSH was absent (blue line), dopamine was oxidized by $\mathrm{CoOOH}$, and the absorbance of the ultraviolet absorption spectrum at $400 \mathrm{~nm}$ became stronger, indicating the successful synthesis of fluorescent polydopamine particles. Meanwhile, in Fig. S6 (SI), it was proved that the fluorescence of the polydopamine could not be influenced by GSH. While the fluorescent polydopamine particles were mixed with GSH, the fluorescence did not decrease, indicating that the decrease in fluorescence intensity was caused by the decrease of the synthesized fluorescent particles rather than the quenching of GSH.

\section{Optimization of sensing conditions}

We optimized the experimental conditions, including the $\mathrm{HCl}$ concentration, $\mathrm{CoOOH}$ concentration and synthesis time of PDA nanoparticles (Fig. S7, SI). The maximal signal could be obtained when $6 \mathrm{mM} \mathrm{HCl}$ and $45 \mu \mathrm{g} \mathrm{mL}^{-1} \mathrm{CoOOH}$ were present. The purpose of adding hydrochloric acid after formation of the fluorescent PDA nanoparticles was to adjust the $\mathrm{pH}$ of the solution to about 2 to lower the polymerization rate of the quinone. As shown in Fig. S7A, when the concentration of $\mathrm{HCl}$ was increased to $6 \mathrm{mM}$, the $\mathrm{pH}$ of the solution was about 2 , and the fluorescence intensity of the PDA nanoparticles was the highest. Therefore, $6 \mathrm{mM} \mathrm{HCl}$ was selected as the optimum condition in subsequent experiments. As shown in Figs. S7B and S7C, it was found that the fluorescence intensity of PDA nanoparticles increased with increasing $\mathrm{CoOOH}$ from 7.5 to $45 \mu \mathrm{g} \mathrm{mL}^{-1}$, but as the concentration of $\mathrm{CoOOH}$ continued to increase, the fluorescence intensity decreased gradually, which may have been caused by
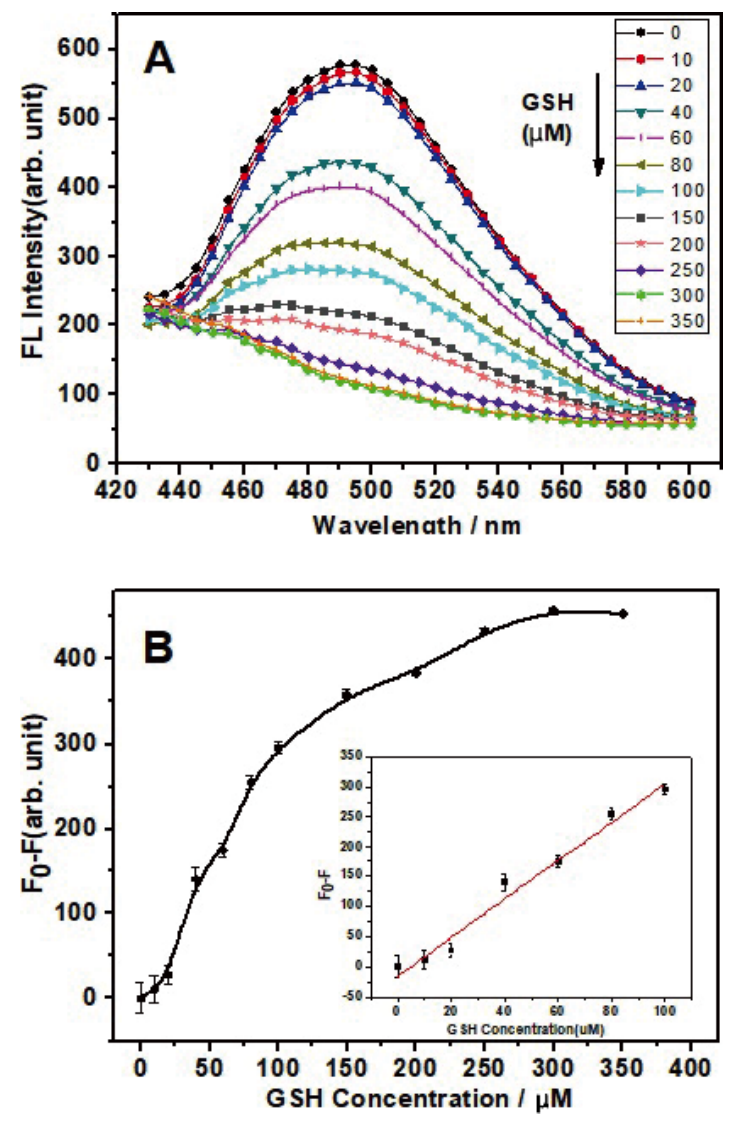

Fig. 3 (A) Responses of the fluorescent polydopamine particles with diverse concentrations of GSH $(0-350 \mu \mathrm{M})$. (B) Relationship between $\left(F_{0}-F\right)$ and $\mathrm{C}_{\mathrm{GSH}}$. Error bars came from three repetitive experiments.

the quenching effect of excess $\mathrm{CoOOH}$ and the formation of large PDA nanoparticles. Meanwhile, we also optimized the reaction time between $\mathrm{CoOOH}$ and dopamine, and when the reaction reached $40 \mathrm{~min}$ the signal achieved the highest level (Fig. S7D). At last, we optimized the reaction time between GSH and $\mathrm{CoOOH}$ (Fig. S8, SI). Once the time reached $20 \mathrm{~min}$, the signal reached a steady state and did not change again after that. We thus chose $20 \mathrm{~min}$ as the reaction time between GSH and $\mathrm{CoOOH}$.

\section{The detection of GSH}

Under the optimized conditions, the working curve was collected. A different concentration of GSH was added into the system, and then reacted for $40 \mathrm{~min}$ (Fig. 3A). As the concentration of GSH increased, the fluorescence intensity gradually decreased. When the GSH concentration exceeded $300 \mu \mathrm{M}$, the fluorescence did not change and reached the platform. The fluorescence decreased values $\left(F_{0}-F\right)$ correlated linearly with the GSH concentrations between 10 and $100 \mu \mathrm{M}$ (Fig. 3B), and the detection limit was $1.93 \mu \mathrm{M}$ (according to $3 \sigma$ rule). These results illustrated that the work was highly sensitive, and provided a fast means for GSH sensing compared with other developed detection platforms (Table S1, SI).

\section{Selectivity analysis}

To evaluate the selectivity of the method before being applied in a real sample, we used a variety of different components as interferences (the concentrations of these interferences were 


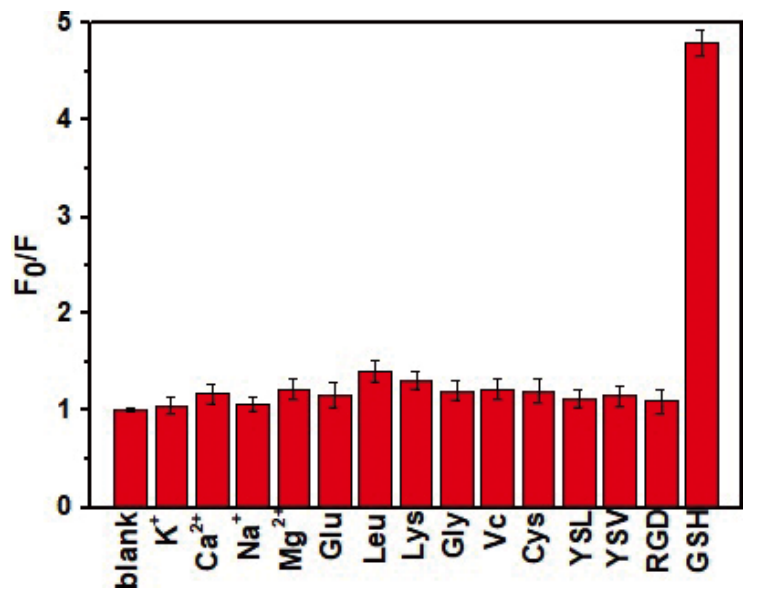

Fig. $4 F_{0} / F$ values in response to GSH and interferents. $\left(F_{0}\right.$, the fluorescence value with no GSH; $F$, the fluorescence value with GSH or other interference species present). Error bars came from three repetitive experiments.

Table 1 GSH detection in blood samples

\begin{tabular}{cccccc}
\hline Sample & $\begin{array}{c}\text { Measured/ } \\
\mu \mathrm{M}\end{array}$ & $\begin{array}{c}\text { Added/ } \\
\mu \mathrm{M}\end{array}$ & $\begin{array}{c}\text { Found/ } \\
\mu \mathrm{M}\end{array}$ & $\begin{array}{c}\mathrm{RSD}, \% \\
(n=3)\end{array}$ & $\begin{array}{c}\text { Recovery, } \\
\%\end{array}$ \\
\hline 1 & 15 & 25 & 41.2 & 4.1 & 103 \\
2 & & 50 & 66.4 & 5.5 & 102.2 \\
3 & & 100 & 113.2 & 3.8 & 98.4 \\
\hline
\end{tabular}

listed in Table S2, SI). It could be seen from Fig. 4 that only when the GSH $(300 \mu \mathrm{M})$ was added, an obvious increase of $F_{0} / F$ was observed, and when other interference components were added to the system, there was no obvious increases. It was worth noting that when cysteine and vitamin $\mathrm{C}$ were at a high concentration, they were able to reduce $\mathrm{CoOOH}$ into $\mathrm{Co}^{2+}$ and cause the fluorescence intensity ratio to increase. ${ }^{35,36}$ However, GSH concentration in human blood was much higher than these interferences, ${ }^{37,38}$ thus demonstrating that the proposed method had a desirable selectivity for GSH.

\section{GSH detection in human blood}

GSH was tested in blood using the assays that we developed to demonstrate the suitability of this protocol. As shown in Table 1, the recovery obtained after adding different amounts of GSH to the blood was between 98.4 and $103 \%$ with the relative standard deviation from 3.8 to $5.5 \%$. From the result, we could see that the PDA system can analyze GSH in real samples.

\section{Conclusions}

In summary, a new platform has been developed to monitor GSH levels. $\mathrm{CoOOH}$ could oxidize dopamine to fluorescent polydopamine particles. Once GSH existed in the system, $\mathrm{CoOOH}$ was reduced to $\mathrm{Co}^{2+}$, and dopamine could not continue to be oxidized to fluorescent PDA particles. In addition, the probe that we constructed enabled the fast and sensitive detection of GSH. We also found that this probe had a good performance in GSH detection in real samples. This reveals its potential value to be applied in biological analysis.

\section{Acknowledgements}

This work was supported by the National Natural Science Foundation of China (No. 21525522 and 21705039), Hunan Provincial Science and Technology Department (No. 2019RS3011), and the Foundation for Innovative Research Groups of NSFC (Grant 21521063).

\section{Supporting Information}

This material is available free of charge on the Web at http:// www.jsac.or.jp/analsci/.

\section{References}

1. S. Lu, Mol. Aspects Med., 2009, 30, 42.

2. (a) D. M. Townsend, K. D. Tew, and H. Tapiero, Biomed. Pharmacother, 2003, 57, 145. (b) J. M. Estrela, A. Ortega, and E. Obrador, Crit. Rev. Clin. Lab. Sci., 2006, 43, 143.

3. U. Sivasankaran, S. Jesny, A. R. Jose, and K. G. Kumar, Anal. Sci., 2017, 33, 281.

4. L. G. Chandrasena, L. D. R. De Silva, K. I. De Silva, P. Dissanayaka, and H. Peiris, Southeast Asian J. Trop. Med. Publ. Health, 2008, 39, 731.

5. J. Q. Huang, J. C. Zhou, Y. Y. Wu, F. Z. Ren, and X. G. Lei, Free Radic. Biol. Med., 2018, 127, 108.

6. S. J. Chinta and J. K. Andersen, Free Radic. Biol. Med., 2006, 41, 1442.

7. E. L. Bettermann, T. J. Hartman, K. A. Easley, E. P. Ferranti, D. P. Jones, A. A. Quyyumi, V. Vaccarino, T. R. Ziegler, and J. A. Alvarez, J. Nutr., 2018, 148, 245.

8. A. K. Sakhi, K. M. Russnes, S. Smeland, R. Blomhoff, and T. E. Gundersen, J. Chromatogr. A, 2006, 1104, 179.

9. G. Y. Shi, J. X. Lu, F. Xu, W. L. Sun, L. T. Jin, K. Yamamoto, S. G. Tao, and J. Y. Jin, Anal. Chim. Acta, 1999, 391, 307.

10. R. Rellan-Alvarez, L. E. Hernandez, J. Abadia, and A. Alvarez-Fernandez, Anal. Biochem., 2006, 356, 254.

11. J. Mendoza, T. Garrido, R. Riveros, and J. Parada, Phytochem. Anal., 2009, 20, 114.

12. Q. Y. Liu, J. Bao, M. Yang, X. J. Wang, S. Y. Lan, C. J. Hou, Y. Z. Wang, and H. B. Fa, Sens. Actuators, B, 2018, 274, 433.

13. H. P. Peng, M. L. Jian, Z. N. Huang, W. J. Wang, H. H. Deng, W. H. Wu, A. L. Liu, X. H. Xia, and W. Chen, Biosens. Bioelectron., 2018, 105, 71.

14. (a) X. Q. Chen, Y. Zhou, X. J. Peng, and J. Yoon, Chem. Soc. Rev., 2010, 39, 2120. (b) P. K. Sudeep, S. T. S. Joseph, and K. G. Thomas, J. Am. Chem. Soc., 2005, 127, 6516. (c) S. Banerjee, S. Kar, J. M. Perez, and S. J. Santra, Phys. Chem. C, 2009, 113, 9659.

15. (a) Y. H. Ahn, J. S. Lee, and Y. T. Chang, J. Am. Chem. Soc., 2007, 129, 4510. (b) L. Yi, H. Li, L. Sun, L. Liu, C. Zhang, and Z. Xi, Angew. Chem. Int. Ed., 2009, 48, 4034. (c) N. Shao, J. Jin, H. Wang, J. Zheng, R. Yang, W. Chan, and Z. Abliz, J. Am. Chem. Soc., 2010, 132, 725.

16. J. K. Jaiswal, H. Mattoussi, J. M. Mauro, and S. M. Simon, Nat. Biotechnol., 2003, 21, 47.

17. I. L. Medintz, H. T. Uyeda, E. R. Goldman, and H. Mattoussi, Nat. Mater., 2005, 4, 435.

18. X. Michalet, F. F. Pinaud, L. A. Bentolila, J. M. Tsay, S. Doose, J. J. Li, G. Sundaresan, A. M. Wu, S. S. Gambhir, and S. Weiss, Science, 2005, 307, 538. 
19. N. L. Rosi and C. A. Mirkin, Chem. Rev., 2005, 105, 1547.

20. X. Sun, S. Yang, M. Guo, S. Ma, M. Zheng, and J. He, Anal. Sci., 2017, 33, 761.

21. D. Liu, X. Pan, W. Mu, C. Li, and X. Han, Anal. Sci., 2019, 35,367

22. Y. Tsuchido, A. Yamasawa, T. Hashimoto, and T. Hayashita, Anal. Sci., 2018, 34, 1125.

23. M. J. Ruedas-Rama, J. D. Walters, A. Orte, and E. A. H. Hall, Anal. Chim. Acta, 2012, 751, 1.

24. O. S. Wolfbeis, Chem. Soc. Rev., 2015, 44, 4743.

25. C. Dai, C. X. Yang, and X. P. Yan, Anal. Chem., 2015, 87, 11455.

26. Y. Zhang, J. M. Liu, and X. P. Yan, Anal. Chem., 2013, 85 , 228.

27. T. T. Chen, Y. H. Hu, Y. Cen, X. Chu, and Y. Lu, J. Am. Chem. Soc., 2013, 135, 11595.

28. Y. Cen, Y. M. Wu, X. J. Kong, S. Wu, R. Q. Yu, and X. Chu, Anal. Chem., 2014, 86, 7119.

29. C. Ma, T. Bian, S. Yang, C. H. Liu, T. R. Zhang, J. F. Yang,
Y. H. Li, J. S. Li, R. H. Yang, and W. H. Tan, Anal. Chem., 2014, 86, 6508.

30. A. Reisch and A. S. Klymchenko, Small, 2016, 12, 1968.

31. Y. Yang, Y. Cen, W. J. Deng, R. Q. Yu, T. T. Chen, and X. Chu, Anal. Methods, 2016, 8, 7199.

32. Y. Cen, J. Tang, X. J. Kong, S. Wu, J. Yuan, R. Q. Yu, and X. Chu, Nanoscale, 2015, 7, 13951.

33. W. Zheng, H. Fan, L. Wang, and Z. Jin, Langmuir, 2015, 31,11671 .

34. X. J. Kong, S. Wu, T. T. Chen, R. Q. Yu, and X. Chu, Nanoscale, 2016, 8, 15604.

35. D. G. He, X. X. Yang, X. X. He, K. M. Wang, X. Yang, X. He, and Z. Zou, Chem. Commun., 2015, 51, 14764.

36. W. Y. Zhai, C. X. Wang, P. Yu, Y. X. Wang, and L. Q. Mao, Anal. Chem., 2014, 86, 12206.

37. Y. H. Wang, K. Jiang, J. L. Zhu, L. Zhang, and H. W. Lin, Chem. Commun., 2015, 51, 12748.

38. L. El-Khairy, P. M. Ueland, H. Refsum, I. M. Graham, and S. E. Volllst, Circulation, 2001, 103, 2544. 